\title{
ACTITUDES DE ESTUDIANTES DE ENFERMERÍA MEXICANOS AL MANEJ AR RESIDUOS PELIGROSOS BIOLOGICO INFECCIOSOS
}

\author{
Atitudes de Estudantes de Enfermagem Mexicanos ao \\ Manipular Resíduos Biológicos Infecciosos Perigosos \\ Attitudes of Students of Nursing to Handle Mexican \\ Biological Infectious Hazardous Waste
}

Micaela Olivos Rubio ${ }^{1}$

Gloria Ángeles Ávila²

Beatriz Arana Gómez ${ }^{3}$

\section{RESUMEN}

El manejo de residuos peligrosos biológico infecciosos es actualmente un problema para los estudiantes de enfermería como integrantes del equipo de salud debido a su capacidad para provocar enfermedades. El propósito fue identificar las actitudes como factor de riesgo para los estudiantes de Enfermería en el manejo de los Residuos Peligrosos Biológico Infeccioso. Estudio de tipo cuanti-cualitativo con enfoque descriptivo transversal observacional. La muestra la constituyeron 403 estudiantes de 1ㄴ,20 y 3er grado de la Facultad de Enfermería durante las prácticas clínicas realizadas en hospitales de la ciudad de Toluca México. Se aplicó al total de estudiantes una escala actitudinal tipo Likert, 25 entrevistas y 12 guías de observación. Teniendo como resultados con respecto a las actitudes que muestran los estudiantes durante sus prácticas clínicas la incertidumbre y temor de contraer alguna enfermedad infectocontagiosa, por no saber manejar estos residuos, indiferencia por no creer que existe riesgo, vergüenza a la crítica, coraje por sufrir accidentes con residuos, arrepentimiento por no realizar adecuadamente la clasificación.

Palavras-chave: Enfermería. Estudiantes. Infección. Residuos Peligrosos. Actitud.

\section{Resumo}

0 manuseio de resíduos biológicos infecciosos é perigoso e é atualmente um problema para os estudantes de Enfermagem como membros da equipe de saúde, em razão da sua capacidade de provocar doenças. 0 propósito foi identificar as atitudes como fator de risco para os estudantes de Enfermagem no manuseio destes resíduos. Estudo do tipo quantiqualitativo, com enfoque descritivo, transversal e observacional. A amostra constituiu-se de 403 estudantes de $10,2 \cong$ e 30 graus, da Faculdade de Enfermagem durante as práticas clínicas realizadas em hospitais da cidade de Toluca - México. Aplicou-se a escala atitudinal, tipo Likert, ao total de estudantes, com 25 entrevistas e 12 roteiros de observação. Obteve-se como resultado que os estudantes nas práticas clínicas sofrem com a incer teza e 0 medo de contrair alguma doença infecto-contagiosa, por não saberem manusear estes resíduos, sentem indiferença por não acreditar que existe risco, vergonha em relação a críticas, coragem por sofrer acidentes com resíduos e arrependimento por não realizarem adequadamente a classificação.

Palavras-chave: Enfermagem. Estudantes. Infecção. Resíduos Perigosos. Atitude.

\begin{abstract}
Nowadays the handling of biological infectious hazardous wastes represents a big deal to Mexican nursing students that are part of a health team since these wastes cause illnesses. The intention was to identify the attitudes as factor of risk for the students of nursing in the Biological Infectious managing of the Dangerous Residues. This is a quantitative study which includes an observant and transversal, descriptive transverse focus. The sample consisted of 403 students of $102-$ and 3 $\because$ year at nursing Faculty during the clinical practices realized in hospitals of Toluca México's city. It was applied to the students an attitude Likert scale, 25 interviews and 12 guides of observation. In regards to the attitudes observed during the clinic practices of students it was remarkable the uncer tainty and fear of catching an infectious and contagious illness because of lack of knowledge, indifference because they think there is no risk, embarrassment when being criticized, annoyance when having accidents with wastes and remorse for nothaving done an adequate classification of waster uncertainty and dread of contracting some disease infectious-contagious, for not being able to handle these residues, indifference for not believing in that risk, shame exists to the critique, courage for having accidents with residues, repentance for not realizing adequately the classification.
\end{abstract}

Keywords: Nurse. Student. Infection. Hazardous waste. Attitude. 


\section{INTRODUCCIÓN}

La contaminación producida por la generación de residuos peligrosos domésticos, Industriales y hospitalarios constituyen el talón de Aquiles de una civilización que ha promovido el crecimiento económico y la industrialización como prototipo de la modernización y el desarrollo económico.

El volumen, mundial de residuos peligrosos está provocando el envenenamiento del planeta y de todos sus ecosistemas, degradando la calidad de vida de millones de seres humanos y provocando serios problemas de salud pública. Por lo cual se determino como prioritaria la eliminación global de los contaminantes en los acuerdos de cooperación ambiental suscritos junto con otras naciones en el marco de las cumbres mundiales sobre el medio ambiente realizadas en Estocolmo y Río de Janeiro Brasil congruentes con los aspectos que se abordan actualmente en la cumbre mundial sobre el desarrollo sostenible realizada en Johannesburgo en la cual manifiesta que la salud depende en último término de la capacidad de controlar la interacción entre el medio físico, el espiritual, el biológico, el económico y el social. Comentando que no es posible un desarrollo equilibrado sin una población saludable ${ }^{1}$

Resaltando el hecho de que la mayoría de las actividades de desarrollo afectan en mayor o menor grado al medio ambiente, lo que, a su vez origina ó incrementa muchos problemas de salud, como es el caso de la gran cantidad de desechos generados por toda la población humana creando grandes problemas de tipo ecológico y daños a la salud, provocando una alteración tanto en morbilidad como en mortalidad, sobre todo aquellos desechos de tipo biológico infecciosos, siendo éstos: materiales de curación que contienen microbios o gérmenes patógenos que han entrado en contacto 0 que provienen del cuerpo de seres humanos y/o animales infectados o enfermos (por ejemplo: sangre y algunos fluidos corporales, cadáveres y órganos extirpados en operaciones), asimismo objetos punzo cortantes (incluyendo agujas de jeringas, material de vidrio roto, entre otros. ${ }^{2}$

Desde hace años se acepta que el riesgo biológico, considerado como exposición accidental es, sin duda, el más frecuente entre los riesgos laborales de los trabajadores de la salud, las inoculaciones parenterales (pinchazos, cortes, rasguños) y el contacto con membranas, mucuosas o piel no intacta (lesiones y dermatitis) con sangre, tejido u otros fluidos corporales potencialmente contaminados; también deben valorarse los contactos con piel intacta en tiempo prolongado con sangre u otros fluidos altamente infecciosos, que impliquen un área extensa, así como el contacto directo con muestras de laboratorio. Y dentro de este grupo las inoculaciones accidentales son las que generan una mayor angustia y percepción de riesgo.
De acuerdo a la NORMA 087-SEMARNAT - SSA1-2002. Los Residuos Peligrosos Biológico Infecciosos RPBI's son aquellos materiales generados durante los servicios de atención médica que contengan agentes biológico-infecciosos y que puedan causar efectos nocivos a la salud y al ambiente ${ }^{3}$

Las instituciones de atención a la salud y de investigación son considerados establecimientos generadores de materiales contaminados por agentes biológico-infecciosos, denominados RPBI's, su manejo y disposición inadecuados, representa un riesgo para la salud del personal que labora en estos sitios, así como para la salud de la población aledaña, ocasionando además el deterioro del medio ambiente. Los microorganismos patógenos, virus, parásitos y priones (estructuras proteicas) son considerados en esta categoría. Para que un microorganismo sea capaz de producir enfermedad, es decir que sea un agente Biológico- Infeccioso debe tener las siguientes características: Tener una concentración suficiente (inóculo), estar en un ambiente propicio (supervivencia) encontrarse en presencia de una vía de entrada en un hospedero susceptible.

Algunos accidentes exponen a los trabajadores a agentes patógenos contenidos en la sangre entre los cuales los más importantes son: virus de la inmunodeficiencia humana (VIH), virus de la hepatitis B (VHB) y de la hepatitis C (VHC), como se reporta en un estudio realizado en el Hospital Universitario "12 de octubre" en Madrid España refiere que en un período de 16 años se declararon 550 accidentes con fuente VIH positiva. La media fue de $34.4 \%$ de accidentes por año. La tasa de exposición fue de 7.5 por 1000 trabajadores al año. El profesional con mayor frecuencia de accidentes fue el personal de enfermería en un $54.4 \%$. La tasa media de exposición fue de $2.6 \%$ camas-año y las zonas anatómicas más afectadas fueron los dedos de la mano en un $75.6 \%{ }^{4}$

En otro estudio realizado en el Hospital Infantil de México "Federico Gómez" sobre la exposición accidental a productos biológicos potencialmente infecciosos en personal de salud. Este reporta que durante cuatro años se presentaron 848 accidentes de trabajo, con un promedio de $29.9 \%$ año-cama. Los eventos se presentaron principalmente en personal de enfermería con 345 episodios $40.6 \%$, seguida de médicos residentes con 220 eventos $25.9 \%$. El mecanismo más frecuente fue el piquete de aquja en 616 ocasiones $72.65 \%$, siendo las manos el sitio anatómico más comúnmente afectado en 667 casos $79.8 \%{ }^{5}$

Con lo expuesto anteriormente se damos cuenta que tanto en México como en España los profesionistas más expuestos a accidentes por exposición con material potencialmente contaminado con RPBI's es el personal de enfermería teniendo en cuenta, que aplica el tratamiento directo a los pacientes a través de procedimientos y técnicas terapéuticas y que de alguna manera tiene mayor contacto físico y emocional.

Según datos del Instituto Nacional de Ecología la infraestructura hospitalaria en México es de 128,620 camas y cada una genera un promedio de cuatro kilogramos de basura por día y si a ese volumen se suman las estimaciones de clínicas 
no registradas, centros de investigación, pequeños consultorios y hasta veterinarias, el total de desechos hospitalarios en el país ascienden a 752 toneladas diarias aproximadamente. ${ }^{6}$

En cumplimiento de los acuerdos internacionales suscritos en México en materia de preservación y cuidado del ambiente, México ha logrado significativos avance, no queriendo decir con esto que la problemática en materia de generación de RPBI's esta resuelta y mucho menos la de contaminación por inadecuado manejo por parte de los integrantes del personal de salud. Lo que se debe resaltar es el hecho que actualmente México cuenta con un marco legal que regula de alguna forma el manejo y la producción de estos residuos en las instituciones de salud entre las que se encuentra: La Ley general del Equilibrio Ecológico y Protección al ambiente, la ley General de Salud, así como las siguientes normas; NOM-052 ECOL93, NOM-087ECOL1995, NOM-87-SEMARNAT-SSA1-2002.

Los RPBI's son un riesgo para la salud de los integrantes del equipo multidisciplinario que labora en el ámbito hospitalario, donde el personal de enfermería no esta exento, además se suman a este riesgo los estudiantes de enfermería que se encuentran en formación y que acuden a realizar prácticas clínicas en las distintas instituciones hospitalarias y se integran a las actividades de atención integral al paciente, lo que hace que se encuentren en contacto directo con los RPBI's Por lo cual el presente estudio se plantea el siguiente objetivo general: Identificar las actitudes que presentan los estudiantes de Enfermería en el manejo de los Residuos Peligrosos Biológico Infecciosos y de forma específica el identificar la importancia para los estudiantes en el manejo de estos residuos, y a su vez determinar si los estudiantes tienen disposición para su cor recta clasificación y los riesgos y efectos que causa un inadecuado manejo de estos residuos. En estudios anteriores se han reportado lesiones en los estudiantes de Enfermería causadas principalmente por agujas hipodérmicas y lancetas contaminadas por sangre humana. ${ }^{7}$ Por lo cual es necesario identificar el riesgo potencial que tienen los estudiantes de enfermería en el manejo de los RPBI, debido a que con frecuencia los coordinadores de práctica clínica mencionan que algunos alumnos se contaminan debido a que presentan actitudes negativas al manejar estos residuos.

\section{METODOLOGÍA}

Estudio de tipo cuanti-cualitativo con enfoque descriptivo transversal observacional. La muestra la constituyeron 403 estudiantes de 1020 y 3er grado de la Facultad de Enfermería durante las prácticas clínicas realizadas en hospitales de la ciudad de Toluca México. Aplicándose al total de alumnos la escala Actitudinal, ${ }^{*}$ tipo Likert 25 entrevistas ${ }^{* *}$ y 12 guías de observación ${ }^{* * *}$

Básicamente la investigación se abordó en tres fases las cuales fueron:

-

- Escala Actitudinal tipo Likert Consiste en un conjunto de ítems presentados en forma de afirmaciones o juicios; a cada uno de los puntos se les asignó un valor numérico ya establecido, para que al final la suma de éstos nos permitiera determinar la relación de los estudiantes ante los RPBI. " Cédula de Entrevista.- Se trató de una conversación para conocer la actitud de los estudiantes mediante una serie de preguntas previamente estructuradas, que sirvieron como guía para la realización de la misma; inicialmente los alumnos a entrevistar se eligieron aleatoriamente; sin embrago, no se pudo lograr de esta manera, ya que algunos alumnos no quisieron colaborar, por tal motivo se contó con el apoyo unicamente de aquellos que sí desearon participar. "'Gúáa de Observación. - La observación se utilizó para describir e identificar de manera sistemática el comportamiento de los estudiantes de Enfer mería ante el manejo, uso, conservación y/o eliminación de los Residuos Biológico Infecciosos.
Fase Teórica: En esta fase se recolectó la información bibliohemerográfica y de fuentes electrónicas, en la Secretaría de Ecología, empresas relacionadas con la recolección y tratamiento de los Residuos Biológico Infecciosos, Secretaria del medio Ambiente y Recursos Naturales del Estado de México (SEMARNAT), elaboración de fichas bibliográficas y de trabajo, para conformar el marco referencial.

Fase Empírica o de Campo: En esta etapa se aprovechó la estancia en prácticas clínicas de los estudiantes en las instituciones hospitalarias de la ciudad de Toluca: Instituto Mexicano del Seguro Social (IMSS), Institución de Seguridad Social al Servicio de los Trabajadores del Estado (ISSSTE), Instituto de Seguridad Social del Estado de México y Municipios (ISSEMyM), Instituto Materno Infantil del Estado de México (IMIEM), Instituto de Salubridad del Estado de México (ISEM). Se realizo la prueba piloto de la escala actitudinal tipo likert en el $10 \%$ de la muestra sujeta ha estudio misma que se capturó mediante la utilización del programa SPSS versión 11.5 , obteniendo una validez de por medio del método estadístico Alpha - Cronbach de 0.66 haciendo las modificaciones necesarias para la aplicación al total de la muestra, así como la ejecución de la guía observacional y entrevista a profundidad ${ }^{8}$

Fase Analítica: En la Escala actitudinal: El análisis de los datos estadísticos se llevo a cabo a través de la de la estadística descriptiva para cada variable, utilizando medidas de tendencia central como la media para la edad, además de relacionar lo anterior con el marco referencial.

Para el análisis de resultados de las entrevistas y Guías de observación; se codificaron e interpretaron los datos, describiendo los contextos, eventos, situaciones y experiencias de los estudiantes sujetos a estudio; además se explicaron sucesos y actitudes que se identificaron durante la observación.

La elaboración de análisis se efectuó mediante la integración del análisis estadístico y el cualitativo, manejando dos núcleos temáticos: Importancia en el manejo de los RPBI's y la identificación que tienen los alumnos hacia estos residuos, con el propósito de respaldar el planteamiento e hipótesis de la investigación.

\section{RESULTADOS E DISCUSIÓN}

La intención primordial de esta investigación es identificar las actitudes que tienen los estudiantes de enfermería en el manejo de los RPBI durante sus práctica-clínicas, teniendo en cuenta que la media de edad de los encuestados fue entre los 20 y 22 años los resultados se presentan en dos núcleos temáticos:

\section{A) Núcleo temático: Importancia en el manejo de Ios RPBI's.}

En cuanto a la escala actitudinal se obtuvo que en relación a la importancia del manejo de RPBI's. El $95.3 \%$ de los estudiantes encuestados consideran importante el manejo de los RPBI mostrando una actitud positiva y de interés no sólo por su salud, sino también por la protección de los pacientes; sin embargo, el $28.5 \%$ los alumnos del tercer grado son los 
que consideran menos importante el manejo de RPBI's; esto debido probablemente a que sólo en la primera práctica se les da información al respecto. ${ }^{9}$ Tomando en cuenta que el proceso de establecer relaciones interpersonales con los demás se basa en la propia opinión; es decir en el yo personal, cuando estas relaciones se han desarrollado en forma saludable contribuyen a lograr una buena opinión, lo que ayuda al estudiante de enfermería a relacionarse satisfactoriamente con el paciente y logra manejar actitudes positivas como: Respeto, independencia, honestidad, libertad, comunicación, confidencialidad, entre otros, fortaleciendo la importancia que se da a la autoprotección personal ante situaciones que pueden comprometer la salud como integrante del equipo de salud. ${ }^{10}$ Lo cual reafirman los estudiantes a través de la entrevista como a continuación se muestra.

El manejo de los RPBI es importante, porque pueden infectar al personal multidisciplinario que trabaja en el hospital, sería indispensable que todos los que trabajamos en el área de la salud conozcamos la norma oficial o el reglamento de cada institución... (E-3)

En cuanto a la clasificación que se le da los RPBI's el $61 \%$ de los estudiantes manifiesta no tener dificultad en depositar adecuadamente estos residuos cuando realizan sus prácticas clínicas, pero el $32.5 \%$ manifiesta temor al manejarlos para su clasificación.

El manejo de residuos peligrosos biológico-infecciosos de acuerdo a la NOM 87 NOM-87-SEMARNAT-SSA1-2002, tiene estipulado para los generadores y prestadores de servicios, además de cumplir con las disposiciones legales aplicables, deben cumplir con las disposiciones correspondientes a las siguientes fases de manejo, según el caso:

a) Identificación de los residuos.

b) Envasado de los residuos generados.

c) Almacenamiento temporal.

d) Recolección y transporte externo.

e) Tratamiento.

f) Disposición final.

En las áreas de generación de los establecimientos generadores, se deberán separar y envasar todos los residuos peligrosos biológico-infecciosos, de acuerdo con sus características físicas y biológicas infecciosas, Durante el envasado, los residuos peligrosos biológico-infecciosos no deberán mezclarse con ningún otro tipo de residuos municipales o peligrosos. ${ }^{2}$

En cuanto a las entrevistas, los estudiantes manifestaron que los RPBI's, se clasifican de acuerdo a la institución en la que se encuentren realizando prácticas, aunque han visto que en la mayoría de los hospitales los manejan de la siguiente manera: existe una bolsa roja donde depositan todo lo que está en contacto con el humano, una bolsa negra o transparente donde van por lo regular envolturas de lo que se ocupa en el hospital, la amarilla que pocas instituciones la manejan y un contenedor de plástico de color rojo para las agujas, otros dicen que en la bolsa roja van todos los residuos no anatómicos, en la bolsa amarilla los anatómicos y los punzocortantes en envases rígidos.

Si, bueno se clasifica de acuerdo a lo que estás manejando, se utiliza la bolsa blanca, roja y la amarilla. En la bolsa blanca se pone todo 10 que son envolturas, por decir toda la basura normal, en la bolsa roja se coloca to do lo que está en contacto con el paciente y en la bolsa amarilla se coloca por decir órganos, jah! y las agujas se colocan en los contenedores rojos (E-18).

En la observación se detectó que los alumnos con una actitud de inseguridad al clasificar los RPBI actuando con nerviosismo y desinterés; como ejemplo, una alumna le daba más impor tancia al procedimiento de enfermería que realizaba, y los residuos que se generaban los acumulaba en la mesa del paciente 0 en la charola de su material, esta alumna se encontraba realizando prácticas en el Hospital del IMSS, en el cual no existen contenedores por cubículo, por lo tanto los trasladaba hasta la Central de Enfermería o el séptico, sin precaución alguna, con el riesgo de pincharse o contaminarse al depositarlos.

También se pudo observar que influye de manera considerable que los alumnos tengan que adaptarse a los recursos con que cuentan las diferentes instituciones hospitalarias, razón por la cual se va creando en ellos una actitud de inseguridad y duda al estar en contacto con estos residuos. De igual manera la clasificación que proporcionan las diferentes instituciones a los RPBI se detectó que es muy variado, incluso dentro de la misma institución, un ejemplo de ello es el hospital del ISEM, en donde se identificó que en los servicios de ginecología y nefrología los contenedores de los residuos no cuentan con las características requeridas e incluso el color de la bolsa es igual para el contenedor de estos y la basura municipal, creándose así una situación de per turbación en los alumnos, ya que al no haber ningún tipo de identificación y diferencia en los contenedores pueden llegar a depositar los RPBI en un contenedor equivocado, mientras que en el hospital del IMIEM se observó que cuentan con los contenedores para residuos no anatómicos (bolsa roja), basura municipal (bolsa blanca) y el contenedor para punzocortantes, sin embargo, esto no ocurre en todos los servicios del hospital, como el servicio de pediatría, donde no hay contenedores por cubículo, sólo se encuentran en la central de enfermeras.

\section{B) Núcleo Temático: Identificación de riesgos en el manejo de RPBI's.}

En cuanto a este núcleo, el $50 \%$ de los estudiantes expresa no tener problemas, mientras que el otro $50 \%$ manifiesto que sí tiene problemas para identificar estos riesgos.

Lo descrito por los alumnos durante las entrevista es, que durante sus prácticas clínicas los estudiantes están más en 
contacto con residuos como secreciones del organismo, los punzocortantes y que por lo general tratan con pacientes a los cuales se les tiene que sacar muestras de sangre, en ocasiones se les realizan lavados bronquiales, lavados gástricos, enemas, canalizaciones, curaciones, colocación de sondas. Tomando en consideración que estos procedimientos deben de realizarse con técnica aséptica para evitar contagio y utilizar una técnica estéril para después depositarios en el lugar donde corresponden.

En práctica hemos tenido contacto casi con todos los RPBI, porque he manejado pacientes que se les tiene que hacer, no sé, sacar sangre, en ocasiones se les tiene que hacer bronco aspiraciones 0 lavados gástricos o enemas, entonces todas esas sondas o catéteres tienen que ser manejados con técnica aséptica para evitar el contagio y utilizar una técnica estéril y no quitarme los guantes hasta que termine de depositar en el lugar donde corresponde y prosigo con el siguiente paciente(E-13).

Con respecto al riesgo que representan los RPBI para la salud de los estudiantes, éstos afirman en su mayoría que sí son un peligro para ellos, mostrando una actitud de interés, poco más de la mitad manifiesta una actitud de enojo al contaminarse con alguno de estos residuos, tomando medidas necesarias, puesto que el $50 \%$ de ellos conoce los efectos que puede tener el mal manejo de los RPBI, ya que han recibido por lo menos una plática de alguna institución al inicio de práctica.

Los estudiantes manifiestan en las entrevistas que es poca la experiencia que tienen en cuanto al manejo de los RPBI, puesto que cuando llegan al hospital se tienen que adaptar a las políticas de la Institución, aún cuando en algunos hospitales existen carteles donde hay información para el manejo de los RPBI.

Los estudiantes exponen que les preocupa contaminarse con los RPBI's, ya que la enfermería es una profesión humanista, pero también con peligro, por eso es necesario saber manejar estos residuos, ya que un mal manejo puede tener consecuencias hasta mortales, afortunadamente los alumnos toman algunas precauciones y medidas necesarias para evitar contaminarse; 10 estudiantes refiere en las entrevistas haber sufrido accidentes con material peligroso 0 contaminado, los accidentes que más predominaron fueron, aunque en muy bajo porcentaje, con agujas hipodérmicas, seguidos con lancetas, igualmente contaminadas, otros accidentes reportados fueron por contacto con material biológico infeccioso; el más prevaleciente fue con sangre humana, para algunos alumnos el haber sufrido un accidente les dejó la enseñanza de no colocar los residuos cuando los contenedores han rebasado su capacidad, pero desafor tunadamente otros piensan que el tener un esquema de vacunación o realizarse una asepsia y antisepsia los excluye de no contraer ningún tipo de enfermedad infectocontagiosa. ${ }^{11}$

En la observación se identificó que algunos alumnos al realizar sus procedimientos de enfermería toman sus precauciones, ya sea lavándose las manos después de cada procedimiento, utilizando guantes, cubre bocas, pero al no contar con los recursos necesarios para clasificar a los RPBI, ellos sólo les proporcionan un manejo de acuerdo a sus posibilidades, ya que en ocasiones tienen que llevar guantes, cubre bocas, botas o gel antiséptico para su protección durante sus prácticas clínicas; en algunas ocasiones sus coordinadores les proporcionan este material, sin embargo, no todos tienen la posibilidad de adquirirlos.

Es evidente que un poco más de la $60 \%$ de los alumnos al contaminarse con los RPBI presentan mayor interés en su salud al informar a las autoridades correspondientes, puesto que las instituciones tienen la obligación de auxiliarlos y proporcionarles tratamiento oportuno, según el artículo $4^{\circ}$ de la Constitución Política de los Estados Unidos Mexicanos, que menciona que toda persona tiene derecho a la protección de su salud, por lo cual la mayoría de los estudiantes manifiesta que al realizar sus procedimientos de enfermería tiene cuidado de no contaminarse. ${ }^{12}$

Los alumnos comentan que durante su formación académica en la Facultad de Enfermería y Obstetricia se les proporciona muy poca información acerca del manejo de los RPBI's, así como sobre las medidas preventivas que deben tomar, tanto para evitar accidentes como después de ocurridos, puesto que el riesgo sigue latente después de prácticas, ya que muchas enfermedades infectocontagiosas se presentan mucho tiempo después del contacto directo con el microorganismo. ${ }^{13}$

\section{CONCLUSIONES}

-En cuanto a la importancia del manejo de los RPBI's, desde el punto de vista actitudinal se identificó que los estudiantes consideran en forma general que es importante el manejo integral de estos residuos.

- Respecto a la clasificación de los RPBI's los estudiantes manifiestan no tener dificultad en depositar adecuadamente estos residuos, detectando también que a algunos estudiantes se le dificulta la clasificación, debido a que durante sus prácticas clínicas tienen confusión por la diversidad de formas de clasificación en las instituciones, y esto hace que su actitud sea de indiferencia al realizar la clasificación.

- Los estudiantes manifiestas un gran interés y disposición en cuanto al manejo de los RPBI's. a pesar de la poca experiencia y falta de algunos conocimientos que tienen en cuanto al manejo de estos.

-Los estudiantes conocen parcialmente los riesgos que puede ocasionar un deficiente manejo de los Residuos Peligrosos Biológico Infecciosos, destacando que un mal manejo puede tener consecuencias mortales.

-Es importante considerar que el interés y disposición que presentan los estudiantes con respecto al manejo de los residuos sean estas actitudes que logren el obtener mayor conocimiento para beneficio en el cuidado al paciente.

-Es importante señalar que más de la mitad de los estudiantes tiene acceso a la información en el manejo de los RPBI's; sin embargo, presentan una actitud de temor al manejarlos durante las prácticas clínicas, por la falta de unificación en el manejo de estos residuos a nivel hospitalario. 
-Se detecto que los estudiantes presentan las siguientes actitudes al manejar los residuos:

Miedo: De contraer alguna enfermedad infectocontagiosa. Incertidumbre: A que hacer en el momento de no saber manejar los RPBI's.

Vergüenza: A ser criticado por no saber cual es la clasificación. Arrepentimiento: Por no realizar adecuadamente la clasificación.

Indiferencia: Al tomar la actitud de "no me pasa nada" si no realizo correctamente la clasificación.

\section{Referências}

1.Cumbre Mundial sobre Desarrollo Sostenible. Johannserburgo Sudáfrica; [on-line] 2006 [citado 24 jun 2007]. Disponible en: http:// agenda21ens.cicese.mx/capitul06. htm.

2.Díaz BF. Los residuos peligrosos en México. [ensayo] México (MX); 1996.

3.México. Secretaría de Gobernación. Diario Oficial de la Federación. 2003.

4.García DC. Accidentes con exposiciones a material biológico contaminado con VIH en trabajadores de un hospital de tercer nivel de atención. Rev Electr Salud Publica [on-line] 2004 [citado 20 enero 2006]. Disponible en: www.scielosp.org/ scielo. php? script $=$ sci_arttext\&pid $=$ S1135-

5.Morales AJ. Frecuencia y mecanismos de exposición accidental a productos biológicos potencialmente infecciosos en personal de salud.Rev Electr Medigraph [on- line] 2006 [citado 12 Oct 2006] Disponible en: scielo.unam.mx/cgi-bin/wxis.exe/iah/?lsisScript=iah/ iah. $x$ is \&base $=$ article\%

6.Comisión Nacional de Ecología (MX). Informe de la situación en materia de equilibrio ecológico y protección al ambiente. México (MX); 1990.
Coraje: Cuando sufre un accidente provocado por el mal manejo de los RPBI's.

-Solo algunos estudiantes presentan desinterés al manejo de los RPBI's influido por la poca información que se les proporciona durante su formación académica dentro de la Facultad, por lo que en muchas ocasiones, aunque conocen los efectos que puede tener un mal manejo de los RPBI's, no les proporcionan la importancia necesaria.

7.Gómez FG. Incidencia de accidentes y factores de riesgo en las prácticas clínicas de los estudiantes de nivel licenciatura. [tesis de licenciatura]. Facultad de Enfermería / Universidad Autónoma del Estado de México. México (MX); 2002.

8. Hernández SP, Fernández CC, Baptista LP. Metodología de la investigación, México (MX): Mac Graw Hill; 2006.

9.Moya M. Psicología social. México (MX): Mac Graw Hill; 1999.

10.Lartigue T. Enfermería una profesión de alto riesgo. México (MX): Plaza Valdés Ed; 1998.

11.Gestal 0Jj. Riesgos laborales del personal de enfermería. México (MX): Mac Graw Hill; 2003.

12. Constitución Política de los Estados Unidos Mexicanos, 5 de febrero de 1917. México (MX): Ed Mexicanos; 2007.

13. Universidad Autónoma del Estado de México. Facultad de Enfermería y Obstetricia. Curriculum de licenciatura con antecedentes de profesional asociado en enfermería general. México (MX); 2001.

14.Hospital Pablo Tobon Uribe. Manual para el Manejo de los Desechos sólidos Hospitalarios. Medellín (CO); 1992.

15.Konning H. Desechos peligrosos y salud en América Latina. Washington (USA): OPS/OMS; 1997. 